error in medical matters which is injurious, but to regard the unacademic with contempt is irrational as well as narrow; successful popular remedies may contain a grain of truth. Homeopathy meant that the medical profession should. avoid heavy, nauseous, and needless drugging. Christian Science indicates that psychology and a knowledge of the working of subconscious thought is needed in medical practice. The catarrh cures preceded laryngology, carcer cures without the knife pointed the need of cancer research and radium study. A prevalence of osteopathy and bone-setting means a need of a more scientific study of physical therapeutics.

\section{Brininal Artixleg.}

\section{PRIMARY SYPHILIS.*}

By C. Guy Lane, M.D., Boston,

Assistant Dermatologist, Massachusetts General Hospital; Assistant Physician, Department of Dermatology, Boston Dispensary.

THE subject of primary syphilis affords an opportunity to emphasize one of the big questions arising in the field of preventive medicine in connection with this disease. The matter of prevention in all its aspects is a subject of first importance, and next in importance comes the early recognition and the care of the early stages in order to prevent later effects. As an introduction to this discussion of the earliest stage of syphilis, it is my intention to emphasize certain modern concepts of this disease as a whole.

In the first place, let me call attention to the concept of syphilis as a general disease, in order to emphasize the fact that it should be treated as such instead of treating merely the local manifestations. In dispensary practice, as well as in private practice, physicians working much with syphilis find that this fact is often lost sight of. Many cases are seen in which the treatment has been continued only until the local condition has disappeared. Certain facts about syphilis contribute to this er. ror. The disease begins as a sharply localized lesion. It appears later in life, often involving a single portion of the body, as a gumma-as a periostitis - aortitis - keratitis, etc. These evidences tend to make us neglect the consid-

* Read before the New Bedford Medical Society, Oct. 11, 1920. eration of this third great plague as a generalized disease. We tend to forget that it is a septicemia, or rather, a bacteremia, at a very early stage in the disease, and that the causative organisms are carried to every nook and cranny in the body, with the ability to lie dor. mant for many years unless entirely eradicated by rational and long continued general treat.ment.

Experimental work by Neisser, in 1911, showed that it was possible to transfer the infection by means of the blood before the appearance of secondary symptoms. The failure of circumcision in the prevention of secondary symptoms has demonstrated the same fact. Still more recent is the evidence presented by a positive Wassermann before the appearance of secondary symptoms. The early invasion of the central nervous system, as Fordyce and cthers have shown by the early positive Wassermann tests obtained on the spinal fluid, is still further evidence of the early infection of the deeper structures of the body. Within the last year or two, at the Rockefeller Institute, ${ }^{1}$ experimental work with rabbits has demonstrated that in-seven days after the local inoculation of spirochetes into the testicles of a rabbit the presence of spirochetes in the blood has been shown, and this in view of the fact that the primary appears at the site of inoculation in the rabbit clinically twelve to fourteen days after inoculation, much the same as in man. Moreover, in other experiments on rabbits, if the inguinal glands are removed forty-eight hours after inoculation of the testicles with spirochetes, and material from these glands injected into other rabbits, the disease will be produced almost constantly. Thus can be seen the early invasion of the blood stream and the reason for the failure of excision of the primary. lesion. The conditions are not exactly the same, of course, but there is no reason to doubt, in view of all the experimental work which has been done, that the course of the disease is much the same in the two species.

Another concept which has been brought to our attention, mostly through the efforts of the Public Health Service and the various state health departments, is the seriousness of the ravages of this disease. Here again the true state of affairs is rather masked. Let me cite an example from our own state. Downing ${ }^{2}$ hasanalyzed the vital statisties for this state for 1912. The records show that only 113 deaths 
are recorded as due to syphilis. He points out, however, that if one considers still births, paresis, tabes, paralysis without cause, softening of the brain, certain cerebral hemorrhages, particularly between twenty and fifty; certain heart and arterial diseases, certain liver diseases, some of the suicides, etc., there are, in round numbers, probably 3,000 deaths, or one in eighteen, from the effects of this discase. This ranks next to tuberculosis, pneumonia, and cancer as causes of deaths. Vedder estimates that almost one-half of all syphilitics eventually succumb as a result of the infection. It is the greatest cause of death of men in the large cities.

In the third place, it is practically impossible to estimate the morbidity and economic loss from syphilis. I know of no way to emphasize properly this conception of the disease. There are so many factors which enter into this phase of the question that only a few can be named: the time lost from work, the lessened efficiency, the expense of doctors and medicine, maintenance of hospitals and dispensaries for their care, the cost of state aid and care, etc., to say nothing of actual suffering and disturbed family affairs. In our own state, according to Williams, ${ }^{3}$ it cost $\$ 40,000$ for 100 men admitted to the Boston State Hospital for syphilitic mental disease, and about 10 per cent. of all cases admitted are syphilitic. It costs a big sum, if one figures it all out. This concept can be emphasized again by calling attention to the fact that there are probably, on a conservative estimate, more syphilities in the United States ${ }^{4}$ than there are people in the New England States, or, that their number is twice as great as the population of our own State of Massachusetts.

The cause of syphilis, the treponema pallida, was discovered by Schaudinn and Hoffmann in 1905, while attempting to prove or disprove the existence of a parasite previously advanced as the causative factor. The organism is spiral, corkscrew-like, with very delicate and perfect spirals. It is very actively mobile, advancin: with a screw-like motion, and at other times exhibiting quick lashing motions in which the two ends are approximated. The individuals vary in length roughly from the diameter of a red blood corpuscle to twice this length. It can be grown in pure culture, produce the same dis ease when inoculated into animals, and be re. covered in pure culture, thus fulfilling Koch's law. The earlier experimental work was in apes and monkeys, but the more recent contributions to our knowledge of the disease are duc to the work with rabbits. Fortunately for the human race, the treponema is not very resistant. It dies very quickly with drying. It does not curl up in the dust or lie about in the corners as does the T.B. bacillus. If kept moist at body temperature, it may live for twentyfour hours, and in tissue it may live for fortyeight hours, and in undisturbed culture, under proper conditions, it may live for one year. It is very susceptible to antiseptics and is killed by 60 degrees Centigrade for five minutes. No spores have been found and it is thought that reproduction takes place by transverse division. Experiments have shown that its resistance to mercury and arsphenamine can be raised, especially its resistance to mercury. This bears out certain clinical evidence that we have to deal at times with a mercury resistant or an arsphenamine resistant type of organism. Further experimental work indicates that there are certain strains of treponema just as there are strains of streptococei. Nichols ${ }^{5}$ has isolated a strain which has produced neurosyphilis in man and consistently produced choroiditis in rabbits. This fact is demonstrated again in the individuals who have only skin lesions, bone lesions, or nerve lesions without other manifestations, and also in the families in whicl.t there are several paretics. It is not yet possible, however, to prophesy, from a study of a treponema or a primary lesion, the type of disease which is apt to follow.

The primary lesion of syphilis, or chancre. appears about three or four weeks after infection. It may appear earlier, or as late as six weeks after inoculation. The term "hard" and "soft," as applied to chancre, ought not to be used. It is far more preferable to use the correct terms, chancre and chancroid. About 9? per cent. ${ }^{6}$ of the initial sores are on the genitals, the other 8 per cent. being scattered over the rest of the body, most frequently on the lips, tongue, throat, and breasts. The contag. iousness of these sores is illustrated by the famous kissing party reported by Schamberg, ${ }^{7}$ in which eight individuals were infected by a chancre of the lip. The typical primary lesion has certain definite characteristics. It is usually a painless lesion. It is indurated, the induration at times being almost cartilaginous in character. It is essentially a papule, dull red in color, sometimes crusted, eroded, or ulcer 
ated, with comparatively little discharge. It may vary much in size and may be single or multiple. Numerous classifications of types of primary lesions are found in textbooks, but the various types usually have certain essential characteristics, as I have said, these character. isties being modified somewhat with the location, and particularly, with the amount and character of the mixed infection. Another. characteristic is the involvement of the neighboring glands. Within two or three weeks these satellite glands become enlarged and hard, but are not particularly painful. The clinical reccgnition of a typical untreated primary lesion is not difficult. In regard to the mixed infections, in the lesions which have been treated, and in extragenital lesions, a diagnosis may often be impossible clinically.

I shall purposely say very little about the clinical differential diagnosis, for I feel that laboratory methods play such an important part in and should be applied to the diagnosis of questionable lesions. A chancroid, even a frankly chancroidal ulcer, ought to be considered as a possible hiding place of treponema, and should be examined with that possibility in mind until such time as the diagnosis of syphilis can positively be disposed of. These ulcers are usually excavated, with undermined edges, are painful, with glands which tend to suppurate, and have a short incubation period. Scabies is characterized by itching, other lesions on the body, and often the typical burrows. I have seen a few cases in which the only lesions were on the penis. Herpes is usually a group of vesicles with some itching or burning, without glandular involvement. A carcinoma appears late in life, develops slowly, is often painful, and may have a pearly border. A late syphilitic ulcer is a slow-growing, sluggish, infected one. It does not usually show the characteristic enlargement of the inguinal glands and may have a history of previous syphilis. In all these diseases, in fact, in any sort of sore on the genitals, the possibility of early syphilis must be considered. Not only that, but in the knowledge of the fact that there are 8 per cent. which are elsewhere on the body, the same consideration must be given to all sluggish ulcerations and slow-healing abrasions and fissures, particularly with a painless enlargement of the nearby glands. Again let me repeat two lessons which should be learned: Any lesion of the genitals may be the abiding place of the preponema pallida; and secondly, it is impossible to make a positive diagnosis in many cases from the clinical appearance alone.

It is in order to avoid the later consequences of syphilis, which I have mentioned in my in. troduction, that I want to lay so much stress on the fact that these cases should be sumbitted to laboratory tests-to a dark-field examination at the earliest possible moment, and in an untreated condition. Let me dwell on this latter point. The cauterization of penile lesions and their treatment with antiseptics before a darkfield is done, that is, before an attempt has been made to ascertain the presence of the organisms of syphilis, is a serious error. It may destroy the chance of making a positive diagnosis of syphilis until the disease has becom? widespread enough to provoke a serological change. It may deprive the individual of the advantage of treatment at the most favorable moment. It may allow him to continue in an infectious condition with the possibility of infecting others. It is far more important that an early syphilis be detected than that a chancroid or a herpes should go untreated fo: twenty-four to forty-eight hours. The former is a generalized disease with far-reaching and late effects, while the latter are localized diseases of comparatively short duration. Therefore, don't cauterize, don't treat with calomel or blchloride or strong antiseptics until a darkfield has been made. And if such application has been made, use a normal salt solution dressing for twenty-four to forty-eight hours and make another examination at the end of that time. The darkfield bears the same relation to syphilis that the culture does to diphtheria, only with the darkfield one has the opportunity to see the actual, living, moving cause of the disease in question. In the use of the darkfield as a diagnostic measure for syphilis in the early stages, and in thus paving the way for prevention of the latter stages by proper treatment, we make a practical and effective contribution to preventive medicine.

There are certain details in the darkfield technic which tend to simplify the process so that I feel it is worth while to review the method of making this examination. I have emphasized its value and I might add that it is worth even more to us in the matter of diag. nosis than the examination of sputum in suspected tuberculosis. The special equipment 
necessary consists of the darktield stage and a source of strong light. A hard rubber "funnel stop" is necessary for insertion into the oil immersion lens if this is to be used. Thin slides: and cover glasses should also be used. As a matter of fact, a piece of blotter the size of a quarter, attached to the under side of an ordinary Abbêl condenser, gives a very satisfactory darkfield. A 150-candlepower, nitrogen lamp, enclosed in an asbestos-lined or metal-lined box, is necessary to produce sufficient light. The most satisfactory lamps, naturally, are those sold by regular instrument makers, such as the Bausch \& Lomb, and the Leitz.

In the matter of obtaining specimens for ex amination, attention to certain details increases the chances of finding the organisms, if pres. ent. I have mentioned the necessity of obtain ing specimens from untreated lesions, if possible. The first step which will aid us is to wash off the surface with 95 per cent. alcohol, rubbing vigorously enough to cause a slight oozing If the lesion is covered with epithelium, it should be scarified with a scalpel. Mop off once or twice with dry gauze to get rid of the excess blood. Experience has shown that pressure tends to force more treponema to the sur. face and thus into the serum. Have the individual squeeze the lesion at the base as hard as possible without causing too much pain. It is very necessary that the microscope be centered by means of the circles on top of the darkfield condenser, that is, these circles should be in th: center of the microscopic field when viewed with the low power lens. Furthermore, it is necessary that a drop of oil be placed on the top of the condenser, sufficient to fill in the space between it and the under surface of the slide, and also that a drop of oil be placed on the surface of the cover glass if the oil immer. sion lens is to be used. I prefer to use the oil immersion, but the high dry lens gives sufficient magnification to make a diagnosis. We hear considerable discussion about various treponema which may be found by this method. In genital lesions it ought to be fairly easy to distinguish between the treponema pallida and the treponema refrigens. The latter organism is shorter, thicker, with smaller and less regular turns, and has not the same character istic motion of the pallida. In the mouth lesions, there is more difficulty. Diagnosis from microscopic evidence alone should be made with hesitation. The surface of mucous lesions must be cleansed thoroughly and an endeavor mad; to obtain material from beneath the surface, in order to avoid contamination with similar organisms found in the mouth.

In the cases which are positive it is needless to say that treatment should be started at once. In the negative ones, further examinations, repeated as the individual circumstances suggest, should be made, using salt solution, as I have indicated before. Such cases should be watched and blood taken for Wassermann examination at the time of the first examination, and again in two to four weeks, or sooner if necessary, in an endeavor to settle the question of syphilitic infection once for all. As I say, it is imperative that this question of syphilis, or not syphilis, be determined at the earliest possible moment in order to give the individual and society every possible advantage of preventing the full development of a serious contagious disease. I believe that a procedure similar to this should be followed in every case of genital lesions, or other suspicious lesions, the details, of course, varying somewhat with the indications in the individual case.

There are other laboratory methods to aid us in the diagnosis of early cases. A very practical aid to the darkfield examination is the puncture, with a sterile hypodermic needle, of a satellite gland or the indurated base of a suspicious lesion. Gentle motion of the syringe, while the needle is within the gland substance, either with or without the injection of a tiny amount of sterile salt solution, will result in the maceration of a certain amount of tissue so that perhaps some cloudy fluid may be drawn up into the needle. This material, expressed on a slide and examined under the darkfield, may show the characteristic spiral organisms and aid in the definite determination of those lesions in which a darkfield examination of the surface is negative.

The serological test, the Wassermann test, does not help us in the first few days, but does stand in the second line of attack, as it were. A positive result is given in about 60 per cent. of the cases within three weeks of the appearance of the primary. This fact emphasizes still more the value of an early darkfield examination as a means of diagnosis before serological change is evident. The value of a single positive Wassermann is often exaggerated. Stand- 
ing alone, with no other evidence, it needs confirmation. In the presence of suspicious signs or symptoms, it becomes a valuable ally in diagnosis. The correct interpretation of results demands a knowledge of the particular laboratory and the sensitiveness of the technic which is being used. The general reliability of the technic in this state has been shown recently in 3000 cases by comparison of serological tests performed in two different laboratories in Boston. ${ }^{8}$ This series has demonstrated a uniformity of results in the two laboratories in over 96 per cent. of the cases on one test. In the great majority of the small number showing different results, later tests showed agreement.

A third method of laboratory diagnosis in early lesions is by culture. Granting that a darkfield is negative, recent reports indicate that it is possible, in properly equipped laboratories, to excise a piece of tissue, plant on a proper media, and recover the organism in twenty-four to forty-eight hours. Suffice it to say that this method is hardly to be used except in the vicinity of especially equipped laboratories. In all our work of diagnosis, lab oratory as well as clinical, we must be guided by reason and judgment, perhaps having an outline or routine to be followed, varying it to fit the individual case under consideration, the main point being, as I have already stated, to settle the diagnosis of syphilis as soon as possible. It is not necessary, however, to carry our zest for laboratory procedure to the extent described in a case reported by Dr. Pusey of Chi cago, of a boy who had had numerous Wassermanns and darkfields, two spinal punctures, a circumcision, and a biopsy, in an attempt to make a diagnosis of syphilis in a case of herpes progenitalis.

In the prognosis of early syphilis, there is abundant reason to believe that our newer methods of treatment will afford to the individua? an absolute cure. Indications are that treatment instituted early in the disease, before the treponema have become widely disseminated in large numbers, will kill off these organisms and prevent them from settling down in those areas in which they are accustomed to localize in the later stages. I believe that early, persistent treatment of these earlier cases practically guarantees the individual from further consequences of this infection.

In the local treatment, the use of calomel ointment or calomel powder is usually sufficient, in addition to the usual regular treatment, which should be started as soon as the diagnosis is made. Let me emphasize the fact that treatment should not be instituted until a diagnosis is positively made. Tentative treatment, or incomplete treatment, may spoil the possibility of making a diagnosis at a very early stage, but, at the same time, no delay should be made in beginning treatment at the earliest possible moment when the diagnosis is certain. In early syphilis, we are confronted with a disease which has the actual living organisms circulating in the blood stream. With this indication for treatment before us, the necessity is seen at once for the introduction of those agencies which will kill off the organisms as quickly as possible with the least damage to human structures. The most powerful spirillicide which we have i.s arsphenamine, the drug which experimental and clinical experience has shown performs this work to the most satisfactory degree that is known at the present time. This drug should be administered intravenously, according to the careful directions which accompany each dose of the drug. Too much attention to detail can not be given to the preparation of the solution. and to the administration. It is necessary that distilled water, and freshly distilled water, be used, and that water of proper temperature should be used, according to the brand of drug employed. The matter of neutralization exactly according to directions, the matter of filtration. the matter of proper tubing, the matter of speed with which the drug is administered, all are matters to be given the utmost consideration. The necessity for perfect sterilization of utensils and the fact that we are dealing with a toxic drug, are factors which should always be borne in mind. It is through the neglect of details in mixing and neutralizing and administering the drug that so many serious mishaps and accidents have arisen. In the administration of arsphenamine the most satisfactory results, in the long run, are obtained by the use of the gravity method, employing a dilution of 0.1 gram to at least 20 c.c. sterile water, using an 18 to 20 gauge needle. A Fordyce needle, or the use of some similar adapter, tends to make the actual insertion of the needle into the vein a much easier process. Let me emphasize again the necessity for care both in the preparation of the solution and in its admin- 
istration, in order to avoid the possibility of un- be given during the period of administration of desired effects. The preparations produced in arsphenamine. It should be administered to this country today are, as a whole, less toxic the toleration of the individual over a period than foreign preparations, and are giving reliable results in the hands of the majority of the men who are using them.

Individuals who are to receive salvarsan should receive a certain amount of preparation. In the first place, they should have a physical examination before treatment. We ought to know their physical condition before, in order to estimate the dose and frequency of the drug. and to be able to anticipate possible serious ef. fects. The urine should be examined; the circulatory system should be examined; and the blood pressure taken, particularly in individuals over forty. History of previous diseases, particularly in regard to the kidney, heart and liver, should be obtained. The patient should be requested not to eat for at least four hour; before the injection and to take a saline laxative, preferably the night following. Both of these measures tend to lessen the possibility of reactions.

There are two usual methods of prescribing arsphenamine. The first includes six or eight weekly, intravenous injections, in doses calculated to the body weight of the individual, giving 0.1 gram for each thirty pounds of body weight. The first injection of a series should not be larger than one-half the dose for that individual in order to avoid the possibility of an idiosyncrasy to arsenic. The other method of treatment, the so-called intensive method, consists of three full doses of arsphenamine at twenty-four hour intervals, the intention being to eradicate all the treponema at this time, if possible. Pollitzer of New York is the champion of this method, and his results tend to bear out his contention. It must be remembered, however, that he is a man of wide experience and uses the utmost care in the preparation and administration of the drug. I, personally, favor a half-way course, particularly in the type of ambulatory cases which we see in the dispensaries and in the office. I feel that an intravenous injection, calculated as above, every three days, for four doses, followed by two to four more at weekly intervals, provides almost as much assurance in the way of treatment, and at the same time is a bit safer to employ with the average individual.

Mercury is indicated in every case. It may of ten to twelve weeks, always remembering the fact that mercury is more toxic to the kidneys than arsphenamine. The injection of a soluble salt every other day, or the injection of an insoluble salt, such as the salicylate, intramuscularly, once a week, are the methods most in use today. The inunction method is slower and dirtier, but at the same time the saturation can be maintained in one to whom the other methods are objectionable. I repeat that in our eagerness to treat properly we should not lose sight of the fact that both arsphenamine and mercury are toxic drugs; that they both cause irritation to kidney epithelium particularly. Careful examination of urine should be made at frequent intervals, and careful notis should be taken of any untoward circumstances arising after treatment, in order to prevent possible bad results. One should be on the watch for early immediate reactions occurring during or just after administration-the type which causes flushing of the face, possible dyspnea, backache, substernal depression, etc. One should be on the watch for cases in which nausea, vomiting, headache, etc., occur for twenty-four to forty-eight hours after several injections. One should be on the watch for later effects, that is, for the beginning of a dermatitis, which may possibly lead to the serious exfoliative type; for a possible beginning jaundice; for a possible polyneuritis; as well as the possible irritation of the kidney during the course of treatment.

After the first course of treatment with arsphenamine and mercury, lasting, as I have said, from ten to twelve weeks, a rest of four to six weeks may come before repeating the same course again. If the Wassermann remains negative during all this period it may be possible to omit a third course of salvarsan; but I believe, at the present day, in the light of experience to date, it is safer for the individual to have his third course of arsphenamine and mercury in order to be sure of being totally free from the disease. It is not possible to treat the disease properly without the use of both drugs. Practically every authority agrees on this point, the main difference coming in their method of use.

Neoarsphenamine as a substitute for arsphen- 
amine is being used much more extensively. The fact that it dissolves readily in cold water, that it does not require neutralization, and that it is less apt to produce reactions, makes it attractive for administration. Neoarsphenamine solutions are toxic when the drug dissolves with difficulty, or when cloudy solutions are employed, or if shaken in the presence of air. It does not hemolyze the red blood cells in the ordinary concentrations. It is, however, less active therapeutically than arsphenamine, and the clinical experience of most authorities favors the use of the latter. The concensus of opinion is that neoarsphenamine when used as a substitute, should be administered oftener or for a longer period to obtain the same therapeutic effect. In early cases of syphilis, Schamberg ${ }^{9}$ commonly administers 0.9 gram three times a week.

In conclusion, I might summarize by saying, that we are dealing with a contagious disease which becomes generalized at a very early period. It has most serious effects on the individual and on society. Proper laboratory facilities are essential for the diagnosis of early syphilis. In the eradication of the disease, our share, as physicians, lies in the furtherance of efforts at prevention, in the recognition of early stages, and in rational, persistent treatment, in order to prevent the disastrous effects of later involvement and of infection of others.

\section{REFERENCES.}

1 Brown, W. H., and Pearce, Louise: A Note on the Disseminatown, W. H., and Pearce, Louise: A Note on the Dissemina tion of Spiroohaeta Pallida from the Primary Focus of

Arch. Derm. and Syph., Vol. ii, p. 470, October, 1920. Downing: Syphilis and Lung Diseases. Bos
SURGICAL JoURNaL, Vol. clxxii, p. 898, 1015.

SURaICAL Jodrnal, Vol. clxxii, p. 898, 1915.

Williams, F. E.: Relation of Alcohol and Syphilis to

4 Stokes: Third Great Plague. Ed. 1, Philadephia, W. B. Saunders Co., 1917, p. 24.

Sichols: 'Observations on a Strain of Spirochaeta Pallida Isolated

from the Nervous System. Jour. Epx. Med., Vol. xix, p. 362, 1914.

- Porter, H. W.: Statistical Study of Extragenital Chancres. Arch. Derm. and Syph., N. S. Vol. i, p. 15, January, 1920

Schamberg, J. F.: An Epidemic of Chancres of the Lip from

Kissing. Jour. A. M. A., Vol. lvii, p. 788, 1911.

Solomon, H. C. Agreement in Results of the Wassermann Re-

action Jour. A. M. A., Vol. lxxiv, p. 788, March 20, 1920.

- Schamberg, J. F.: Arsphenamine versus Neo-Arsphenamine. Jour.

A. M. A., Vol. lxxiii, p. 1883, Dec. 20, 1919

\section{RECTAL ABISCESSES AND THEIR TREATMENT.}

By T. Chitrenden Hill, M.D., Boston.

THE simplicity of the incision of an abscess as a surgical procedure and the frequency with which abscesses in the perianal region are met have led to minimizing the importance of their treatment. Except in relation to their location. it is true that they differ in no important particular from abscesses in other situations. Their location, however, makes the subject an important one for consideration, for every rectal abscess is a potential fistula.

\section{ETIOLOGY.}

Like all other abscesses, rectal abscesses arise from direct introduction of bacteria. This may take place either through the skin of the perianal region, which is very rich in hair follicles and sebaceous glands, or through some lesion within the rectum, such as an ulcer, abrasion, or fissure.

Pathologists tell us, and indeed it is a wellrecognized fact, that infective bacteria are present in the superficial layers of the skin all over the body. Bacteriological examination of the anal skin in health shows many varieties of pus. producing organisms, and, as both within and without the rectum they are exceedingly numerous, it naturally follows that the perianal region is a favorite site for abscesses. The question of the most common infective agents, however, has never been settled, as bacteriological studies of the pus are beset with great diffculties and a pure culture of any organism is seldom found. The staphylococcus and streptococcus are commonly present; the B. coli is nearly always found and is the cause of the very foul odor so frequently associated with them. Some infections take place through the blood stream,-notably, those following injuries to the parts and those of a tubercular type.

A primary tubercular abscess is most uncommon, infection being more frequently secondary, gaining entrance through swallowed sputum, or from the pus of a tubercular lesion of the intestinal tract. Even such infections must be comparatively rare, however, as I am told that rectal abscesses are not especially numerous in tuberculosis hospitals. Nevertheless, the question whether abscesses and fistulae are not, as a rule, tubercular in origin is frequently asked. To my mind this tubercular theory, so popular among the profession, has erroneously become the commonly accepted conception through the frequency with which such abscesses result in fistulae through improper treatment.

\section{TREATMENT.}

The rapid burrowing of pus about the muscular structures which control the important. function of defecation, if not recognized early 